\title{
Reflexões sobre a logística verde na redução dos impactos ambientais
}

\author{
Green Logistics considerations in assuaging environmental impacts
}

Tiago Henrique de Paula Alvarenga ${ }^{\dagger *}$, Carlos Manuel Taboada Rodriguez ${ }^{\ddagger}$

Como citar esse artigo. Alvarenga, THP; Rodriguez, CMT. Reflexões sobre a logística verde na redução dos impactos ambientais. Revista Teccen. 2018 Jan/Jun.; 11 (1): 47-53.

\begin{abstract}
Resumo
O tema logística verde tem se tornado um "hot topic" na atualidade devido as crescentes demandas por cuidados ambientais nas atividades produtivas. Isso se dá, em grande parte, pelo fato de a maioria dos produtos consumidos em regiões mais desenvolvidas terem seus materiais de recurso na maioria dos casos fornecidos por regiões distantes. Portanto, as atividades logísticas têm recebido muita atenção no que concerne às exigências ambientais. As atividades logísticas são fontes significativas de poluição ambiental e emissões de gases do efeito estufa. Dessa forma, o objetivo deste artigo foi realizar um estudo teórico sobre a Logística Verde, elencando a evolução temporal da logística até ao enfoque ambiental de suas atividades. Na revisão bibliográfica focou-se também em identificar os conceitos essenciais sobre a Logística Verde e as suas atividades que podem ser utilizadas como indicadores. Os resultados demonstraram que a logística passou por uma evolução gradativa, sendo que a Logística Verde está recebendo a atenção de grandes empresas (IKEA e XEROX) na atualidade e que as atividades de logística verde podem ser utilizadas como indicadores para mensurar a diminuição dos impactos ambientais.

Palavras-Chave: Logística; Logística Verde; Atividades de Logística Verde.
\end{abstract}

\begin{abstract}
Green logistics theme has become a hot topic today due to the growing demands for environmental care in productive activities. This is due in large part to the fact that most products consumed in more developed regions have their resource materials in most cases provided by distant regions. Therefore, logistic activities have received a lot of attention regarding environmental requirements. Logistic activities are significant sources of environmental pollution and greenhouse gas emissions. In this way, the objective of this article was to carry out a theoretical study on the Green Logistics, listing the temporal evolution of logistics to the environmental focus of its activities. In the bibliographic review it was also focused on identifying the essential concepts about Green Logistics and its activities that can be used as indicators. The results showed that logistics has undergone a gradual evolution, and Green Logistics is receiving the attention of large companies (IKEA and XEROX) currently and that green logistics activities can be used as indicators to measure the reduction of environmental impacts.

Keywords: Logistics; Green Logistics; Green Logistics Activities.
\end{abstract}

\section{Introdução}

A temática logística verde tem se tornado um “hot topic" na atualidade devido as crescentes demandas por cuidados ambientais nas atividades produtivas. Isso se dá, em grande parte, pelo fato de a maioria dos produtos consumidos em regiões mais desenvolvidas terem seus insumos na maioria dos casos fornecidos por regiões distantes (Ubeda, Arcelus \& Faulin, 2011; Lai \& Wong, 2012), fazendo com que as atividades logísticas recebam muita atenção no que concerne às exigências ambientais (Richardson, 2001).
A Logística Verde preocupa-se em produzir e distribuir bens de forma sustentável, levando em conta fatores ambientais e sociais. Nela os objetivos não se referem apenas ao impacto econômico das políticas logísticas na organização que as realiza, mas também aos efeitos mais amplos sobre a sociedade, como os efeitos da poluição sobre o ambiente. As atividades de Logística Verde incluem a medição do impacto ambiental das diferentes estratégias de distribuição, a redução do consumo de energia nas atividades logísticas, a redução dos resíduos e a gestão do seu tratamento (Sbihi \& Eglese, 2010). Esse gerenciamento verde da

Afiliação dos autores: † Programa de Pós-Graduação em Engenharia de Produção e Sistemas da Universidade Federal de Santa Catarina - UFSC

* Email para correspondência: thpalvarenga@hotmail.com 
logística possibilita a conservação dos recursos naturais, a redução de resíduos e ainda atende as expectativas sociais de proteção ambiental (Lai \& Wong, 2012).

A logística clássica e os modelos tradicionais voltados para produção e distribuição concentram-se na minimização de custos sujeitos a restrições operacionais, deixando de lado as questões ambientais. Assim, a crescente demanda ambiental define que as práticas logísticas atuais não satisfarão as demandas ambientais do futuro (Sbihi \& Eglese, 2010). A necessidade de uma transformação logística diz respeito não apenas ao fornecimento de produtos ou serviços ecológicos aos clientes, mas também ao fluxo logístico global de itens do berço ao túmulo, juntamente com as atividades de logística reversa (Zhou, Cheng \& Hua, 2000).

A globalização da produção tem destacado a importância de um gerenciamento de logística verde na prevenção de danos ambientais causados pelas atividades de fabricação e distribuição de produtos (Lai \& Wong, 2012). Isso tem feito com que muitas organizações avaliem suas pegadas de carbono para que o impacto ambiental de suas atividades possa ser monitorado. Os governos estão a considerar metas de redução de emissões e outras medidas ambientais. Há, portanto, um crescente interesse em Logística Verde por governos e pelas próprias organizações (Sbihi \& Eglese, 2010).

Dessa forma, o objetivo deste estudo foi realizar uma revisão bibliográfica acerca da temática da Logística Verde, bem como identificar atividades de Logística Verde que possam ser utilizadas como indicadores em pesquisas futuras como em surveys, estudos de caso dentre outras modalidades de pesquisa. Para tanto, foi realizado uma revisão bibliográfica em periódicos nacionais e estrangeiros, livros e documentos internacionais sobre a Logística Verde e suas atividades.

\section{Metodologia}

Foi realizado uma revisão bibliográfica acerca da logística verde. Para a realização desta pesquisa foram consultados periódicos nacionais e estrangeiros, livros e publicações internacionais. No que tange a revisão bibliográfica, essa é descrita por Marconi e Lakatos (2008), como a pesquisa ao acervo já publicado sobre a temática em estudo, sendo os exemplos mais comuns periódicos, teses e livros. Os autores defendem que o objetivo desse tipo de pesquisa é possibilitar ao pesquisador um contato com o material já publicado para a construção de conclusões inovadoras. Assim, o procedimento metodológico utilizado no presente trabalho foi a revisão bibliográfica. Dessa forma, a revisão bibliográfica buscou encontrar estudos que fornecessem os primórdios sobre os estudos da logística e logística verde; os conceitos essenciais sobre logística verde e as atividades de logística verde (indicadores).

\section{Logística e Logística Verde}

Ao longo da história da humanidade, guerras são vencidas e perdidas por forças e capacidades logísticas ou pela falta delas. Argumenta-se que a derrota dos britânicos na Guerra da Independência norte-americana pode-se ser largamente atribuída à falha de logística. O exército britânico na América dependia quase que por completo da Inglaterra para obter suprimentos. No auge da guerra, havia 12 mil tropas no exterior e a maior parte não só tinha de estar equipada, mas alimentada (Christopher, 2014).

Na Segunda Guerra Mundial, a logística também desempenhou papel importante. A invasão da Europa por tropas aliadas foi um exercício bastante qualificado na área de logística, como também foi a derrota da Alemanha no deserto africano. No entanto, embora os generais desde épocas remotas tenham compreendido o papel crucial da logística, estranhamente só no passado recente as organizações empresariais reconheceram o impacto vital que a gestão da logística pode ter na conquista da vantagem competitiva (Christopher, 2014).

É nesse cerne que o campo teórico da logística se concentra, buscando compreender como a administração pode prover melhor nível de rentabilidade nos serviços de distribuição aos clientes e consumidores, através de planejamento, organização e controle efetivos para as atividades de movimentação e armazenagem que visam facilitar o fluxo de produtos. A concepção logística de agrupar conjuntamente as atividades relacionadas ao fluxo de produtos e serviços para administrá-las de forma coletiva é uma evolução natural do pensamento administrativo (Ballou, 2015).

A logística empresarial é um campo em expansão com nítidos benefícios para as decisões estratégicas da alta administração. Entretanto, não era esse o caso há décadas atrás, o reconhecimento e crescimento da área de logística foi gradativo e evolutivo (Ballou, 2015). Nessa evolução, há uma nítida pulverização de atividades inerentes a logística no início da segunda metade do século XX, que segue uma lógica de integração (Møller, 1995), e que alcança a demanda ambiental representada pela logística verde na atualidade (Sbihi \& Eglese, 2010; Lai \& Wong, 2012). A figura 1 representa esta evolução.

O serviço de logística em uma cadeia de suprimentos envolve planejamento, projeto, implementação e gerenciamento de fluxo e armazenamento de materiais, e troca de informações para suportar funções específicas como aquisição, distribuição, gerenciamento de inventário, embalagem 


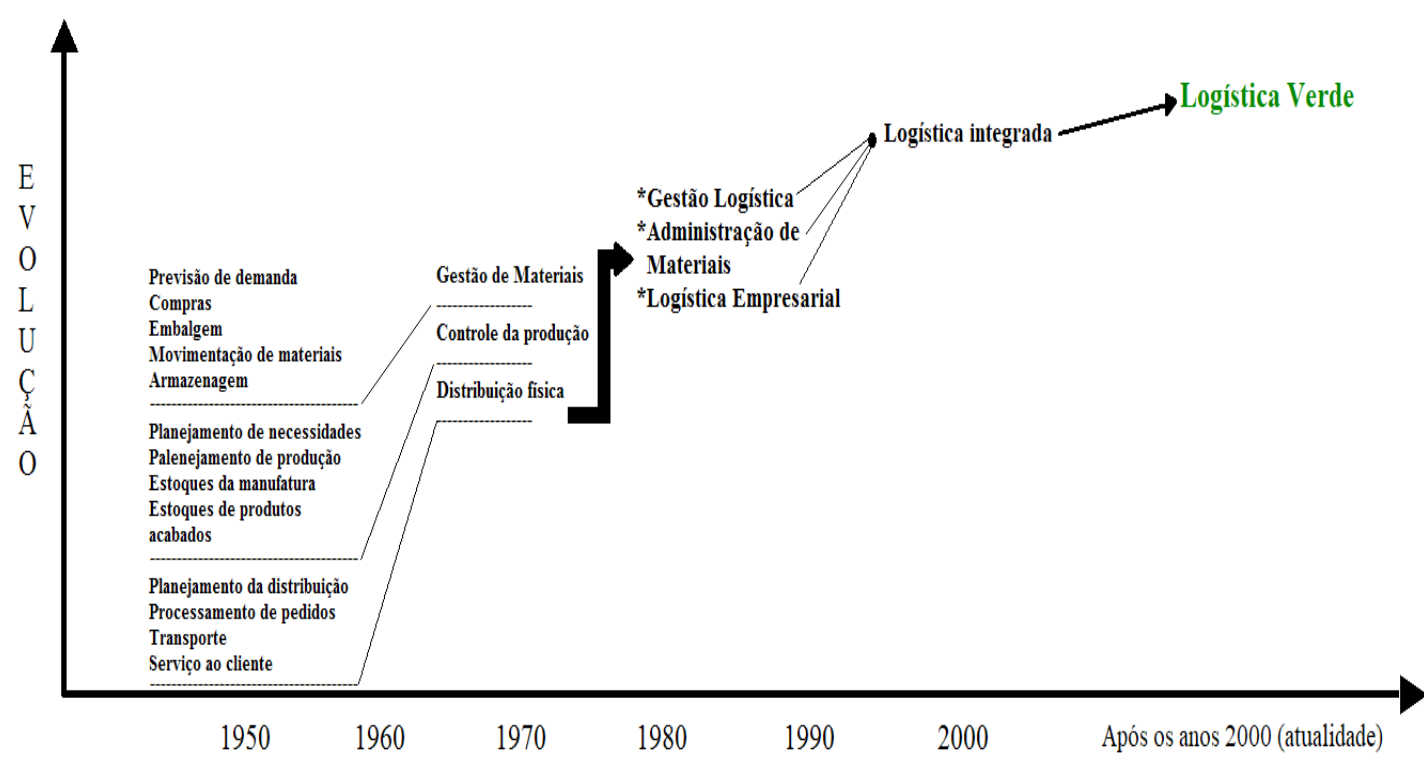

Figura 1. Evolução da logística. Fonte: Adaptado de Møller (1995); Sbihi \& Eglese (2010); Lai \& Wong (2012).

e fabricação (Bowersox \& Closs, 2011).

Esse conjunto de atividades vitais presente no setor de serviços logísticos desempenha um papel fundamental na cadeia de suprimentos. Nos últimos anos esse setor tem atraído atenção tanto de pesquisadores como de profissionais devido ao seu forte impacto negativo ao meio ambiente (Isaksson \& Huge-Brodin, 2013).

As atividades de logística são fontes significativas de poluição ambiental e emissões de gases do efeito estufa, que têm impactos nocivos sobre a saúde humana e a qualidade dos ecossistemas. Portanto, clientes e governos exigem que as empresas reduzam os impactos ambientais (por exemplo, emissões de carbono) de suas atividades (Tsai \& Hung, 2009; Fichtinger et al., 2015).

O movimento em prol da logística verde surgiu no final do século XX e início do século XXI, sendo que vários fatores foram os responsáveis pelo início dessa cinesia. Entretanto, para fins de exemplificação, fatores como a crescente poluição ambiental decorrente da emissão dos gases gerados pela combustão de combustíveis fósseis; a contaminação dos recursos naturais como consequência de cargas desprotegidas, tais como caminhões com produtos químicos que se acidentam e contaminam rios e o vazamento de diversos produtos contidos através do rompimento de diques utilizados para armazenagem de resíduos de atividades produtivas (mineração e celulose) são devidamente recordados para justificar o movimento em prol da logística verde (Donato, 2008).

Devido ao fato da logística verde ser um campo de estudo recente, esta tem atraído a atenção de pesquisadores, pelo seu importante papel no gerenciamento da cadeia de suprimentos. Ao se comprar com o modelo de logística tradicional, é identificado que apenas o objetivo econômico é considerado, mas na logística verde, objetivos sociais e ambientais são considerados além do objetivo econômico (Rabbani, Farrokhi-Asl \& Asgarian, 2017).

Anteriormente, os sistemas de logística se concentraram principalmente no objetivo de aumentar a eficiência das atividades da indústria em relação ao cronograma e aos lucros. No entanto, a atmosfera atual de crescente preocupação com os impactos ambientais introduziu o conceito de logística verde como base para o desenvolvimento de métodos que podem reduzir os impactos ambientais dos sistemas logísticos (Küçükoğlu et al., 2013).

Ela é um dos subcomponentes vitais do processo de Gestão da Cadeia de Suprimentos Verde (Green Supply Chain Management), que é altamente requerida nos últimos anos em virtude da concorrência, globalização, demanda dos clientes e exploração de novos mercados (Isaksson et al., 2011). As práticas verdes responsáveis pelo sucesso da Gestão da Cadeia de Suprimentos Verde, tem, na logística verde um dos seus principais alicerces, ao lado do desenvolvimento de produtos ecológicos, das compras verdes, das práticas de produção ecológica, do uso de tecnologias mais limpas, do gerenciamento verde e do marketing verde (Luthra, Garg \& Haleem, 2014).

A Logística Verde em si trata de questões ambientais relacionadas ao transporte sustentável, manipulação e armazenamento de materiais perigosos, controle de estoque, armazenagem, embalagem e decisões de alocação de localização de instalações que visam reduzir as pegadas de carbono (Murphy, Poist \& Braunschweig, 1996). A este respeito, muitas empresas, 
a exemplo da IKEA e a Xerox, aplicaram uma série de iniciativas verdes para implementar práticas ecológicas na sua rede logística (Tsai \& Hung, 2009).

Autores como Sbihi e Eglese (2010); Kutkaitis e Župerkienė (2011) defendem que a logística verde está associada ao desenvolvimento sustentável. É um processo que engloba ações da organização, para criar um sistema global de logística eficiente e ambientalmente amigável, de forma a garantir o uso eficiente de energia; a conservação dos recursos; a eliminação de resíduos; a melhoria da produtividade do trabalho; a redução do impacto negativo da organização sobre o meio ambiente e o aumento da competitividade da organização.
$\mathrm{Na}$ percepção abrangente da logística verde há também posicionamentos que incluem a logística reversa como uma atividade de logística verde (Sbihi \& Eglese, 2010; Ying \& Li-Jun, 2012). Tendo em conta que o conceito de logística verde está relacionado ao desenvolvimento sustentável, pode-se afirmar que o conceito também se baseia em três níveis equivalentes: econômico, ecológico e social. Isso prova que a implementação do conceito de logística verde em uma determinada empresa deve ser apoiada pelos princípios de responsabilidade econômica, ambiental e social (Vasiliauskas, Zinkevičiūtė \& Šimonytė, 2013; Lozano, 2012). A figura 2 apresenta essa relação.

Sob o enfoque ambiental, a logística verde

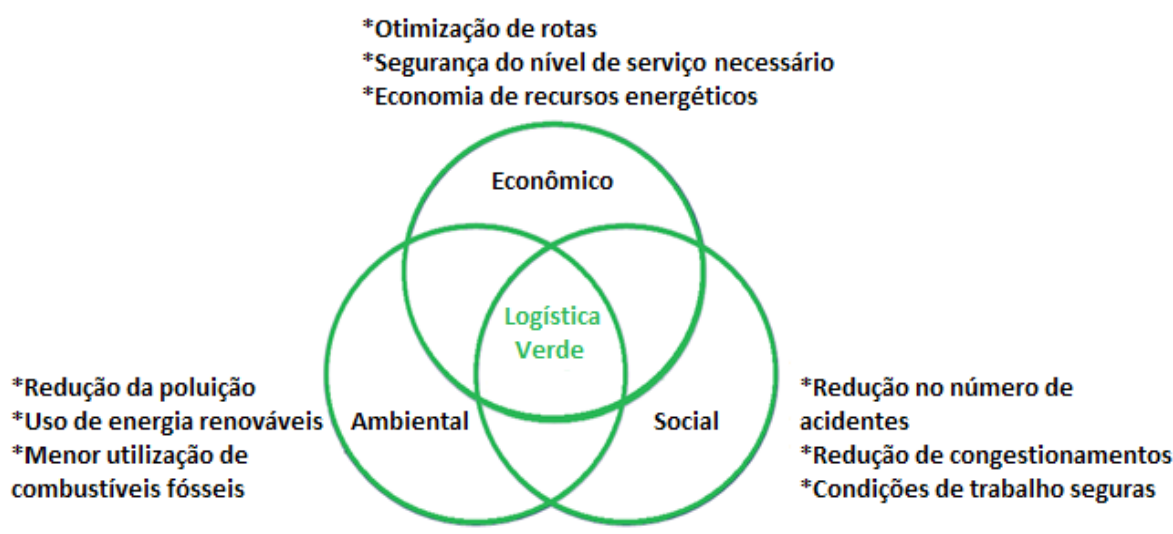

Figura 2. Relacionamento entre a Logística Verde e Desenvolvimento Sustentável. Fonte: Adaptado de Vasiliauskas, Zinkevičiūtė e Šimonytė (2013).

concentra-se na filtragem de emissões, na redução do consumo de energia e nos recursos naturais, na redução dos resíduos e na otimização da exploração de materiais. As práticas verdes comumente usadas no setor de logística incluem a eliminação de resíduos de forma responsável, a compra de produtos ecológicos, o consumo de energia, a redução de resíduos sólidos, utilização de métodos de transporte mais limpos e embalagens/recipientes recicláveis. Assim, uma empresa de logística pode adotar várias práticas verdes para responder a uma variedade de questões ambientais ao mesmo tempo (Gonzalez-Benito; Gonzalez-Benito, 2006).

As exigências legais, o controle dos custos, as expectativas sociais e as demandas dos clientes são incentivos importantes para uma empresa decidir adotar atividades voltadas para a logística verde (Murphy; Poist, 2003). Em suma, a logística verde preocupa-se com a integração dos aspectos ambientais na gestão logística, levando em conta o meio ambiente em cada processo de tomada de decisão (Srivastava, 2007).
Ela reflete a capacidade organizacional de conservar fontes, reduzir o desperdício, melhorar a eficiência operacional e satisfazer as expectativas sociais para a proteção ambiental. Mais do que atividades internas, a exemplo do desenvolvimento de produtos e processos, o gerenciamento de fluxos de produtos físicos é considerado essencial para a conservação do meio ambiente (Lai \& Wong, 2012).

\section{Atividades de Logística Verde (Indicadores)}

Baseado em autores identificados na literatura as atividades de logística verde foram divididas em três categorias, sendo elas: Embalagem verde, transportes verdes e armazenagem e construções verdes. Essa categorização das atividades de logística verde, bem como cada atividade facilitam o entendimento e a sua respectivautilizaçãocomoindicadoresparaamensuração no que concerne as atividades que possam amenizar os impactos ambientais das atividades logísticas. Para 
tanto, foi investigado dentro da literatura existente esses indicadores bem como os seus respectivos autores. $\mathrm{O}$ quadro 1 , apresenta os indicadores de logística verde identificados nesse trabalho:

Quadro 1 - Indicadores de Logística Verde.

Quadro 1. Indicadores de Logística Verde.

\begin{tabular}{|c|c|}
\hline Autores & Embalagem Verde \\
\hline $\begin{array}{c}\text { Lau (2011); Zhu e Sarkis (2004); Zhu, Sarkis e Lai (2013); } \\
\text { Gonzalez-Benito e Gonzalez-Benito (2006); Luthra et al. (2011) }\end{array}$ & $\begin{array}{l}\text { *Utilização de materiais ecológicos } \\
\text { em embalagens }\end{array}$ \\
\hline $\begin{array}{c}\text { Lau (2011); Zhu e Sarkis (2004); Zhu, Sarkis e Lai (2013); } \\
\text { Gonzalez-Benito e Gonzalez-Benito (2006); Luthra et al. (2011) }\end{array}$ & $\begin{array}{l}\text { *Uso de design ecológico em } \\
\text { embalagens }\end{array}$ \\
\hline $\begin{array}{l}\text { Lau (2011); Zhu, Sarkis e Lai (2013); Olugu, Wong e Shaharoun } \\
\text { (2010), Luthra et al. (2011) }\end{array}$ & $\begin{array}{l}\text { *Uso de tecnologias mais limpas em } \\
\text { embalagens }\end{array}$ \\
\hline $\begin{array}{l}\text { Lau (2011); Zhu e Sarkis (2004); Zhu, Sarkis e Lai (2013); } \\
\text { Gonzalez-Benito e Gonzalez-Benito (2006); Luthra et al. (2011); } \\
\text { Kleindorfer et al. (2005); Fleischmann et al. (2001) }\end{array}$ & $\begin{array}{l}\text { *Utilização de materiais de } \\
\text { embalagem reciclados }\end{array}$ \\
\hline $\begin{array}{l}\text { Lau (2011); Zhu, Sarkis e Lai (2013); Kleindorfer et al. (2005); } \\
\text { Fleischmann et al. (2001) }\end{array}$ & $\begin{array}{l}\text { *Recolha de resíduos de materiais de } \\
\text { embalagem utilizados por clientes para } \\
\text { reciclagem }\end{array}$ \\
\hline Autores & Transportes verdes \\
\hline $\begin{array}{c}\text { Rostamzadeh et al. (2015); Perotti et al. (2012); Lau (2011); } \\
\text { Shah (2010); Faruk et al. (2002), Gonzalez-Benito and Gonzalez- } \\
\text { Benito (2006), Lin e Ho (2008), Jumadi e Zailani (2010), Lieb e } \\
\text { Lieb (2010), Léonardi e Baumgartner (2004) }\end{array}$ & $\begin{array}{l}\text { *Otimização na utilização de veículos } \\
\text { energeticamente eficientes }\end{array}$ \\
\hline $\begin{array}{c}\text { Rostamzadeh et al. (2015); Perotti et al. (2012); Lau (2011); } \\
\text { Shah (2010); Faruk et al. (2002), Gonzalez-Benito and Gonzalez- } \\
\text { Benito (2006), Lin e Ho (2008), Jumadi e Zailani (2010), Lieb e } \\
\text { Lieb (2010); Léonardi e Baumgartner (2004) }\end{array}$ & $\begin{array}{l}\text { *Otimização do processo de } \\
\text { distribuição através de uma melhor } \\
\text { rota e agendamento }\end{array}$ \\
\hline Lau (2011); Léonardi e Baumgartner (2004) & $\begin{array}{l}\text { *Uso de tecnologias verdes menos } \\
\text { nocivas ao ambiente dentro do } \\
\text { transporte }\end{array}$ \\
\hline $\begin{array}{l}\text { Rostamzadeh et al. (2015); Shah (2010); Léonardi e Baumgartner } \\
\text { (2004) }\end{array}$ & $\begin{array}{l}\text { *Motivar uma ecocondução para } \\
\text { diminuir o consumo de combustível }\end{array}$ \\
\hline $\begin{array}{l}\text { Rostamzadeh et al. (2015); Perotti et al. (2012); Shah (2010); } \\
\text { Faruk et al. (2002), Gonzalez-Benito and Gonzalez-Benito (2006), } \\
\text { Lin e Ho (2008), Jumadi e Zailani (2010), Lieb e Lieb (2010) }\end{array}$ & *Utilização de combustíveis verdes \\
\hline Autores & Armazenagem e construções verdes \\
\hline $\begin{array}{c}\text { Perotti et al. (2012); } \\
\text { Murphy e Poist (2000); Hervani et al. (2005); Lin e Ho (2008); } \\
\text { Lieb e Lieb (2010); Jumadi and Zailani (2010) }\end{array}$ & $\begin{array}{l}\text { *Materiais de construção oriundos de } \\
\text { processos ecológicos }\end{array}$ \\
\hline $\begin{array}{c}\text { Perotti et al. (2012); } \\
\text { Murphy e Poist (2000); Hervani et al. (2005); Lin e Ho (2008); } \\
\text { Lieb e Lieb (2010); Jumadi and Zailani (2010) }\end{array}$ & *Construções com isolamento térmico \\
\hline $\begin{array}{c}\text { Perotti et al. (2012); } \\
\text { Murphy e Poist (2000); Hervani et al. (2005); Lin e Ho (2008); Lieb } \\
\text { e Lieb (2010); Jumadi and Zailani (2010) }\end{array}$ & $\begin{array}{c}\text { *Construções com iluminação } \\
\text { natural }\end{array}$ \\
\hline $\begin{array}{c}\text { Perotti et al. (2012); } \\
\text { Murphy e Poist (2000); Hervani et al. (2005); Lin e Ho (2008); Lieb } \\
\text { e Lieb (2010); Jumadi and Zailani (2010) }\end{array}$ & $\begin{array}{l}\text { *Sistemas de iluminação } \\
\text { energeticamente eficientes }\end{array}$ \\
\hline $\begin{array}{l}\text { Perotti et al. (2012); } \\
\text { Murphy e Poist (2000); Hervani et al. (2005); Rizzo (2006); Lin e } \\
\text { Ho (2008); Lieb e Lieb (2010); Jumadi and Zailani (2010) }\end{array}$ & $\begin{array}{l}\text { *Equipamentos eficientes } \\
\text { manuseio de materiais }\end{array}$ \\
\hline $\begin{array}{c}\text { Perotti et al. (2012); } \\
\text { Murphy e Poist (2000); Hervani et al. (2005); Rizzo (2006); Lin e } \\
\text { Ho (2008); Lieb e Lieb (2010); Jumadi and Zailani (2010) }\end{array}$ & $\begin{array}{l}\text { *Utilização de fontes alternativas de } \\
\text { energia como painéis solares }\end{array}$ \\
\hline
\end{tabular}




\section{Considerações finais}

A Logística Verde é um campo em ascensão para que as organizações obtenham melhorarias em seu desempenho ambiental, bem como capturar as necessidades dos clientes interessados em práticas ambientais que possam minimizar a degradação do meio ambiente. Para tanto, a inclusão de atividades de logística verde dentro do ambiente da logística se torna premente para o sucesso dessa intenção. A utilização de indicadores baseados em atividades de logística verde propicia mensurar os impactos ambientais das atividades logísticas.

Como contribuição esse trabalho entrega um conjunto de atividades de logística verde (indicadores) que possam ser utilizadas em pesquisas futuras e também no ambiente empresarial e industrial.

\section{Referências}

Ballou, R. H. (2015). Logística empresarial: transporte, administração de materiais e distribuição física. São Paulo: Atlas.

Bowersox, D. J., Closs, D. J. (2011). Logística empresarial: o processo de integração da cadeia de suprimentos. São Paulo: Atlas.

Christopher, M. (2014). Logística e gerenciamento da cadeia de suprimentos. São Paulo: Cengage Learning.

Donato, V. (2008). Logística Verde. Rio de Janeiro: Editora Ciência Moderna Ltda.

Faruk, A.C., Lamming, R.C., Cousins, P.D. and Bowen, F. E. (2002). Analyzing, mapping, and managing environmental impacts along supply chains. Journal of Industrial Ecology, 5 (2), 13-36.

Fichtinger, J., Ries, J. M., Grosse, E. H. and Baker, P. (2015). Assessing the environmental impact of integrated inventory and warehouse management. International Journal of Production Economics, 170, 717-729.

Fleischmann, M., Buellens, P., Bloemhof-Ruwaard, J., and Van Wassenhove, L. N. (2001). The impact of product recovery on logistics network design. Production and Operations Management, 10(2), 156-173

Gonzalez-Benito, J., Gonzalez-Benito, O. (2006). The Role of Stakeholder Pressure and Managerial Values in the Implementation of Environmental Logistics Practices'. International journal of production research, .44(7), 1353-1373

Hervani, A. A., Helms, M.M. and Sarkis, J. (2005). Performance measurement for green supply chain management. Benchmarking: An International Journal, 12(4), 330-353.

Isaksson, K., Bjorklund, M., Evangelista, P. and Huge-Brodin, M. (2011). The challenge and adoption of green initiatives for transport and logistics service providers. In: The 16th Annual LRN Conference 7-9 September, Southampton, United Kingdom, 2011, pp. 1e10.

Isaksson, K. and Huge-Brodin, M. (2013). Understanding efficiencies behind logistics service providers' green offerings. Management Research Review, 36(3), pp.216-238.

Jumadi, H. and Zailani, S. (2010). Integrating Green Innovations in Logistics Services Towards Logistics Services Sustainability: A Conceptual Paper. Environmental Research Journal, 4(4), pp.261-271.

Kleindorfer, P., Singhal, K. and Wassenhove, L. (2009). Sustainable Operations Management. Production and Operations Management, 14(4), pp.482-492.

Küçükoğlu I., Ene S., Aksoy A. and Öztürk, N. (2013). A Green Capacitated
Vehicle Routing Problem with Fuel Consumption Optimization Model. International Journal of Computational Engineering Research, 3(7), pp.1623.

Kutkaitis, A. and Župerkienè, E. (2011). Darnaus vystymosi koncepcijos raiška uosto logistinèse organizacijose. Management theory and studies for rural business and infrastructure development, 2(26), pp.130-137.

Lai, K. and Wong, C. (2012). Green logistics management and performance: Some empirical evidence from Chinese manufacturing exporters. Omega, 40(3), pp.267-282.

Lau, K. H. (2011). Benchmarking green logistics performance with a composite index. Benchmarking: An International Journal, 18(6), pp.873896.

Léonardi, J. and Baumgartner, M. (2004). CO2 efficiency in road freight transportation: Status quo, measures and potential. Transportation Research Part D: Transport and Environment, 9(6), pp.451-464.

Lieb, K. and Lieb, R. (2010). Environmental sustainability in the third-party logistics (3PL) industry. International Journal of Physical Distribution \& Logistics Management, 40(7), pp.524-533.

Lin, C. Y. and Ho, Y. H. (2008). An empirical study on logistics service providers' intention to adopt green innovations. Journal of technology management \& innovation, 3(1), pp.17-26.

Lozano, R. (2012). Towards better embedding sustainability into companies' systems: an analysis of voluntary corporate initiatives. Journal of Cleaner Production, 25, pp.14-26.

Luthra, S., Garg, D. and Haleem, A. (2014). Empirical Analysis of Green Supply Chain Management Practices in Indian Automobile Industry. Journal of The Institution of Engineers (India): Series C, 95(2), pp.119-126.

LUTHRA, S.; KUMAR, V.; KUMAR, S.; HALEEM, A. (2011). Barriers to implementing green supply chain management in automobile industry using interpretative structured modeling techniques: an Indian perspective. Journal of Industrial Engineering and Management, 4(2), pp. 231-257.

Marconi, M. A. and Lakatos, E. M. (2008). Técnicas de pesquisa. São Paulo: Atlas.

Martinsen, U. and Huge-Brodin, M. (2014). Environmental practices as offerings and requirements on the logistics market. Logistics Research, 7(1), pp. 1-22

Møller, C. (1995). Logistics Concept Development - Towards a Theory for Designing Effective Systems. Aalborg University.

Murphy, P. R. and Poist, R. F. (2000). Green logistics strategies: an analysis of usage patterns. Transportation Journal, 40(2), pp. 5-16.

Murphy P. R. and Poist, R. F. (2003). Green perspectives and practices. Supply Chain Management: An International Journal, 8(2), pp.122-131.

Murphy, P., Poist, R. F. and Braunschweig, C. D. (1996). Green logistics: comparative views of environmental progressives, moderates and conservatives. Journal of Business Logistics, 17(1), pp.191-211.

Olugu, E., Wong, K. and Shaharoun, A. (2010). A Comprehensive Approach in Assessing the Performance of an Automobile Closed-Loop Supply Chain. Sustainability, 2(12), pp.871-889.

Perotti, S., Zorzini, M., Cagno, E. and Micheli, G. (2012). Green supply chain practices and company performance: the case of 3PLs in Italy. International Journal of Physical Distribution \& Logistics Management, 42(7), pp.640672

Rabbani, M., Farrokhi-Asl, H. and Asgarian, B. (2016). Solving a bi-objective location routing problem by a NSGA-II combined with clustering approach: application in waste collection problem. Journal of Industrial Engineering International, 13(1), pp.13-27.

Richardson, H. (2001). Logistics in reverse. Industry Week, 250(6), pp.3739.

Rizzo, J. (2006). "Logistics distribution \& warehousing 2006: green building: a new priority", area developments, Disponível em $<\mathrm{http}: / / \mathrm{www}$ areadevelopment.com/specialpub/ldw06/greenbuilding. $\operatorname{shtml}$ ? Page $=1>$ Acesso em: 13 jan. 2018. 
Rostamzadeh, R., Govindan, K., Esmaeili, A. and Sabaghi, M. (2015).

Application of fuzzy VIKOR for evaluation of green supply chain

management practices. Ecological Indicators, 49, pp.188-203.

Sbihi, A. and Eglese, R. (2010). Combinatorial optimization and Green Logistics. Annals of Operations Research, 175(1), pp.159-175.

Shah, N. (2010). Green Purchasing: The issue of Responsible Supply chain Management for Improving The environmental Performance. Disponível em <http:/www.hshieldsconsulting.com/downloads/green_purchasing.pdf $>$ Acesso em: 13 jan. 2018.

Srivastava, S. (2007). Green supply-chain management: A state-of-the-art literature review. International Journal of Management Reviews, 9(1), pp.5380

Tsai, W. and Hung, S. (2009). A fuzzy goal programming approach for green supply chain optimisation under activity-based costing and performance evaluation with a value-chain structure. International Journal of Production Research, 47(18), pp.4991-5017.

Ubeda, S., Arcelus, F. and Faulin, J. (2011). Green logistics at Eroski: A case study. International Journal of Production Economics, 131(1), pp.44-51.

Vasiliauskas, A. V., Zinkevičiūtè, V. and Šimonytė, E. (2013). Implementation of the concept of green logistics reffering to it applications for road freight transport enterprises. Business: Theory and practice/verslas: teorija ir praktika, 14(1), pp. 43-50.

Ying, J. and Li-jun, Z. (2012). Study on Green Supply Chain Management Based on Circular Economy. Physics Procedia, 25, pp.1682-1688.

Zhou, Z., Cheng, S. and Hua, B. (2000). Supply chain optimization of continuous process industries with sustainability considerations. Computers \& Chemical Engineering, 24(2-7), pp.1151-1158.

Zhu, Q. and Sarkis, J. (2004). Relationships between operational practices and performance among early adopters of green supply chain management practices in Chinese manufacturing enterprises. Journal of Operations Management, 22(3), pp.265-289.

Zhu, Q., Sarkis, J. and Lai, K. (2013). Institutional-based antecedents and performance outcomes of internal and external green supply chain management practices. Journal of Purchasing and Supply Management, 19(2), pp.106-117. 